\title{
Montage: a grid-enabled engine for delivering custom science-grade mosaics on demand
}

G. Bruce Berriman, Ewa Deelman, John C. Good, Joseph C. Jacob, Daniel S. Katz, et al.

G. Bruce Berriman, Ewa Deelman, John C. Good, Joseph C. Jacob, Daniel S. Katz, Carl Kesselman, Anastasia C. Laity, Thomas A. Prince, Gurmeet Singh, Mei-Hu Su, "Montage: a grid-enabled engine for delivering custom science-grade mosaics on demand," Proc. SPIE 5493, Optimizing Scientific Return for Astronomy through Information Technologies, (16 September 2004); doi: 10.1117/12.550551

Event: SPIE Astronomical Telescopes + Instrumentation, 2004, Glasgow, United Kingdom 


\title{
MONTAGE: A GRID ENABLED ENGINE FOR DELIVERING CUSTOM SCIENCE-GRADE MOSAICS ON DEMAND
}

\author{
G. Bruce Berriman ${ }^{\dagger a}$, Ewa Deelman ${ }^{\mathrm{b}}$, John Good ${ }^{\mathrm{a}}$, Joseph Jacob ${ }^{\mathrm{c}}$, Daniel S. Katz ${ }^{\mathrm{c}}$, Carl Kesselman ${ }^{\mathrm{c}}$, \\ Anastasia Laity ${ }^{\mathrm{a},}$ Thomas A. Prince ${ }^{\mathrm{d}}$, Gurmeet Singh ${ }^{\mathrm{b}}$, Mei-Hu Su \\ ${ }^{a}$ Infrared Processing and Analysis Center, California Institute of Technology \\ ${ }^{\mathrm{b}}$ Information Sciences Institute, University of Southern California \\ ${ }^{\mathrm{c}}$ Jet Propulsion Laboratory, California Institute of Technology \\ ${ }^{\mathrm{d}}$ Division of Physics, Mathematics and Astronomy, California Institute of Technology
}

Keywords: Astronomical image mosaics, image reprojection, data access, grid computing.

\begin{abstract}
This paper describes the design of a grid-enabled version of Montage, an astronomical image mosaic service, suitable for large scale processing of the sky. All the re-projection jobs can be added to a pool of tasks and performed by as many processors as are available, exploiting the parallelization inherent in the Montage architecture. We show how we can describe the Montage application in terms of an abstract workflow so that a planning tool such as Pegasus can derive an executable workflow that can be run in the Grid environment. The execution of the workflow is performed by the workflow manager DAGMan and the associated Condor-G. The grid processing will support tiling of images to a manageable size when the input images can no longer be held in memory. Montage will ultimately run operationally on the Teragrid. We describe science applications of Montage, including its application to science product generation by Spitzer Legacy Program teams and large-scale, all-sky image processing projects.
\end{abstract}

\section{INTRODUCTION}

Astronomy has a rich heritage of discovery from image data collections that cover essentially the full range of the electromagnetic spectrum. Image collections in one frequency range have often been studied in isolation from those in other frequency ranges. This a consequence of the diverse properties of the data collections themselves - images are delivered in different coordinate systems, map projections, spatial samplings and image sizes, and the pixels themselves are rarely co-registered on the sky. Moreover, the spatial extent of many astronomically important structures, such as clusters of galaxies and star formation regions, is substantially greater than those of individual images.

Astronomy thus has a need for image mosaic software that delivers science-grade mosaics from multiple image data sets as if they were single images with a common coordinate system, map projection etc. That is, the software must preserve the astrometric and photometric integrity of the original data, and rectify background emission from the sky or from the instrument using physically based models. The Montage project [1] will provide the astronomer with the tools needed to build mosaics in Flexible Image Transport System (FITS) [2] format, including support for all common astronomical coordinate systems, all World Coordinate System (WCS) map projections [3], arbitrary image sizes (including full-sky images) and rotations, and user-specified spatial sampling.

Montage has been designed as a scaleable, portable tool kit that can be used by astronomers on their desktops for science analysis, integrated into project and mission pipelines, or run on computing grids to support large-scale product generation, mission planning and quality assurance. It will be deployed operationally on the Distributed Terascale Facility (Teragrid) [4] and be accessible to astronomers through existing astronomy portals. In its initial deployment, Montage will serve images from the 2 Micron All Sky Survey (2MASS) [5], Digital Palomar Observatory Sky Survey

† gbb@ipac.caltech.edu; phone 1626 395-1817; fax 1626 397-7354; http://irsa.ipac.caltech.edu; Caltech Mail Stop 100-22, Pasadena, CA 91125. 
(DPOSS) [6] and Sloan Digital Sky Survey (SDSS) [7]. It can therefore be considered an enabling technology, in that the mosaics it generates will widen avenues of astronomical research, and be a valuable tool in mission planning and quality assurance, including:

- Deep source detection by combining data over multiple wavelengths

- Predictions of source counts and wavelength extrapolations of fluxes

- Spectrophotometry of each pixel in an image

- Position optimization with wavelength

- The wavelength dependent structure of extended sources

- Image differencing to detect faint features

- Discovering new classes of objects

A previous paper described Montage as part of the architecture of the National Virtual Observatory [8]. This paper has two aims: to describe the architecture and performance of Montage, and to demonstrate how the toolkit is finding application in astronomy, especially in the generation of mosaics of Spitzer Space Telescope data.

\section{THE ARCHITECTURE AND PERFORMANCE OF MONTAGE}

\subsection{Architectural Components}

Montage employs the following four steps to compute a mosaic:

- Re-projection of input images to a common spatial scale, coordinate system, and WCS projection

- Modeling of background radiation in images to achieve common flux scales and background levels by minimizing the inter-image differences

- Rectification of images to a common flux scale and background level

- Co-addition of re-projected, background-corrected images into a final mosaic

Montage accomplishes these computing tasks in independent modules, written in ANSI C for portability. This "toolkit" approach controls testing and maintenance costs, and provides considerable flexibility to users. They can, for example, use Montage simply to re-project sets of images and co-register them on the sky, or implement a custom background removal algorithm without impact on the other steps, or define a specific processing flow through custom scripts.

\subsection{Serial Processing of Images}

The first public release of Montage [9], version 1.7, supports serial processing of images, with processing of the four steps described above controlled through a set of simple executives. It has been written in ANSI C for portability, and does not use shared memory. It has only been rigorously tested on machines running Red Hat Linux, but has been successfully run under Solaris and Mac OS X, among others.

The first release emphasized accuracy over speed. In order to support the broadest range of applications, the basic Montage reprojection and image flux redistribution algorithm works on the surface of the celestial sphere. All pixel vertices from both input and output images are projected onto this sphere; if necessary, a coordinate system transform is applied to the input pixel vertices to put their sky coordinates in the same frame as the output. Then, for overlapping pixels, the area of overlap (in steradians) is determined. This overlap, as a fraction of the input pixel area, is used to redistribute the input pixel "energy" to the output pixels.

In this way, total energy is conserved for those input pixels which do not extend beyond the bounds of the output image area. Even when a pixel has "undefined" vertices, such as at the boundaries of an Aitoff all-sky projection,, the same process can be applied by determining an edge pixel's outline on the sky, described in the general case as a spherical polygon. The co-addition engine then creates the final mosaic by reading the reprojected images from memory and weighting each pixel's flux by total input area. 
This approach is completely general and preserves the fidelity of the input images. A comparison of sources extracted from the mosaics with the Sextractor source extraction program shows that, in general, Montage preserves photometric accuracy to better than $0.1 \%$ and astrometric accuracy better than 0.1 of a pixel, for the $10 \mathrm{WCS}$ projections subjected to rigorous testing [10]. Generality in reprojection is achieved at the expense of processing speed. For example, reprojection of a $512 \times 1024$ pixel 2MASS image takes 100 seconds on a machine equipped with a 2.26-GHz Intel processor and 1 GB memory, running Red Hat Linux 8.0.

Two further drawbacks inherent in this distribution of the software are that the maximum image mosaic size is limited by the available computer memory, and co-addition of flux in the reprojected pixels only supports weighting by area coverage.

The Montage team has taken advantage of the software's modular design to address these limitations in a new distribution, version 2.0, that is currently undergoing testing and is scheduled for release in late-Summer 2004. Coaddition has been redesigned to overcome the limitations of memory and weighting just described, and the use of a dedicated module allowed the redesign to proceed without impact on the design of performance improvements for reprojection. These performance improvements have taken two forms:

- development of custom, fast reprojection algorithms applicable to commonly used astronomical projections; these algorithms bypass projection of pixels on to a sphere, and transform input pixel flux directly into output pixel space

- exploitation of the parallelization inherent in the design; many of the steps needed to compute a mosaic can be performed in parallel.

The next three subsections describe these design improvements.

\subsection{A General Co-addition Algorithm for Montage}

The limitations of the available memory on the processing machine have been simply overcome by reading the reprojected images one line at a time from files that reside on disk. Assuming that a single row of the output file does not fill the memory, the only limitation on file size is that imposed by the file system. Images of up to 6 GB have been built with the new software. The algorithm has also been developed further to support quite general co-addition methods. For each output line, mAdd determines which input files will be contributing pixel values, and opens only those files. Each contributing pixel value is read from the flux and area coverage files, and the value of each of these pixels is stored in an array until all contributing pixels have been read for the corresponding output row. This array constitutes a "stack" of input pixel values; a corresponding stack of area coverage values is also preserved. The contents of the output row are then calculated one output pixel (i.e., one input stack) at a time, by averaging the flux values from the stack. Different algorithms to perform this average can be trivially inserted at this point in the program. The greater flexibility of the new software comes at the modest expense of $30 \%$ in speed.

Currently, Montage supports mean and median co-addition, with or without weighting by area. The mean algorithm (default) accumulates flux values contributing to each output pixel, and then scales them by the total area coverage for that pixel. The median algorithm ignores any pixels whose area coverage falls below a specific threshold, and then calculates the median flux value from the remainder of the stack. This median input pixel is scaled by its corresponding area coverage, and written as the output pixel. If there are no area files, then the algorithm gives equal weight to all pixels. This is valuable for science data sets where the images are already projected into the same pixel space (e.g., MSX). An obvious extension of the algorithm is to support outlier rejection, and this is planned for a future release as an enhancement.

\subsection{Performance Improvements Through Custom Reprojection Algorithms}

In its general form, the Montage reprojection algorithm transforms pixel coordinates in the input image to coordinates on the sky and then transforms that location to output image pixel space. Under certain circumstances, this can be replaced by a much faster algorithm which uses a set of linear equations (though not a linear transform) to transform directly from 
input pixel coordinates to output pixel coordinates. This alternate approach is limited to cases where both the input and output projections are "tangent plane" (gnomonic, orthographic, etc.), but since these projections are by far the most common, it is appropriate to treat them as a special case.

This "plane-to-plane" approach is based on a library developed at the Spitzer Science Center [11]. When both images are tangent plane, the geometry of the system can be viewed as in Figure 1, where a pair of gnomonic projection planes intersect the coordinate sphere. A single line connects the center of the sphere, the projected point on the first plane and the projected point on the second plane. This geometric relationship results in transformation equations between the two planar coordinate systems that require no trigonometry or extended polynomial terms. As a consequence, the transform is a factor of thirty or more faster than using the normal spherical projection formulae.

A bonus to the plane-to-plane approach is that the computation of pixel overlap is much easier, involving only clipping constraints of the projected input pixel polygon in the output pixel space.

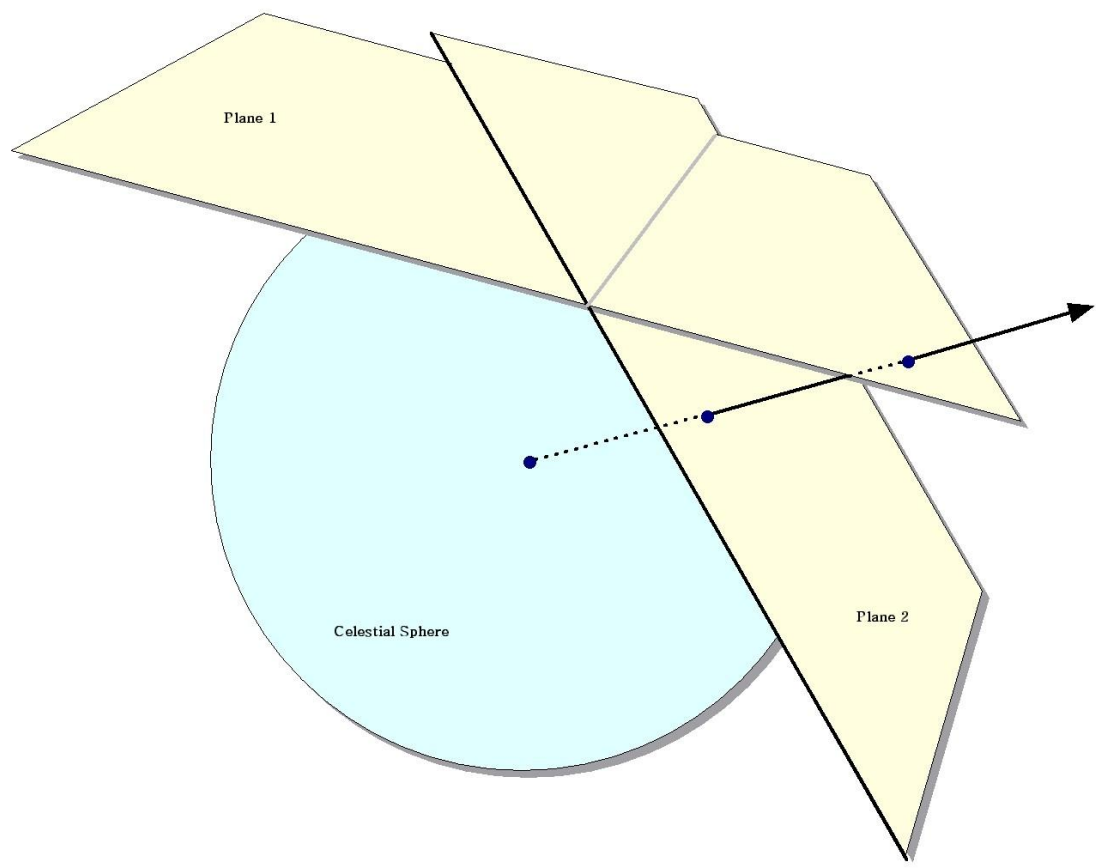

Figure 1: The Principle of Plane-to-plane Reprojection

This approach excludes many commonly-used projections such as "Cartesian" and "zenithal equidistant," and is essentially limited to small areas of few square degrees. Processing of all-sky images, as is almost always the case with projections such as Aitoff, generally requires the slower plane-to-sky-to-plane approach.

There is, however, a technique that can be used for images of high resolution and relatively small extent (up to a few degrees on the sky). Rather than use the given image projection, we can often approximate it with a very high degree of accuracy with a "distorted" Gnomonic projection. In this case, the pixel locations are "distorted" by small distances relative to the plane used in the image projection formulae. A distorted space is one in which the pixel locations are slightly offset from the locations on the plane used by the projection formulae, as happens when detectors are slightly misshapen, for instance. This distortion is modeled by pixel-space polynomial correction terms which are stored as parameters in the image FITS header.

While this approach was developed to deal with physical distortions caused by telescope and instrumental effects, it is applicable to Montage in augmenting the plane-to-plane reprojection. Over a small, well-behaved region, most 
projections can be approximated by a Gnomonic (TAN) projection with small distortions. For instance, in terms of how pixel coordinates map to sky coordinates, a two-degree "Cartesian" (CAR) projection is identical to a TAN projection with a fourth-order distortion term to within about a percent of a pixel width. Figure 2 shows this in exaggerated form for clarity, with the arrows showing the sense of the distortion.

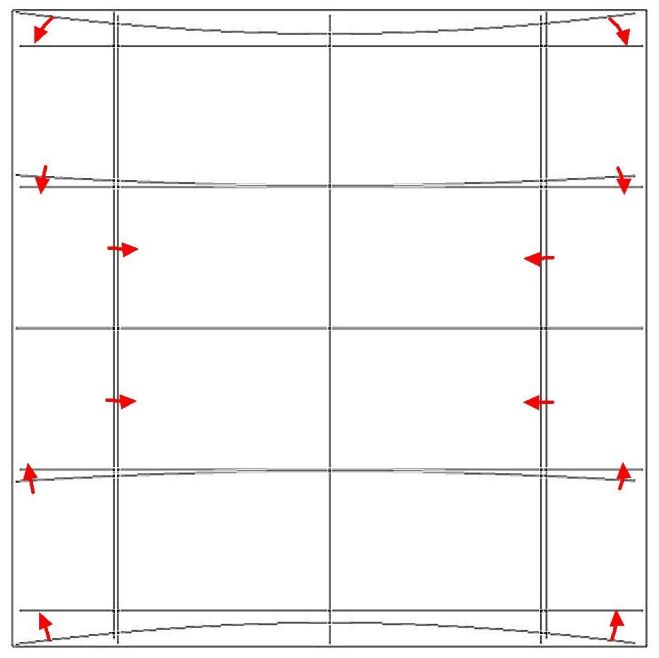

Figure 2: Representation of a WCS projection as a distorted Gnomonic (TAN) projection, exaggerated for clarity. The arrows indicate the sense of the distortions.

In the above example, the curved coordinate grid is an undistorted TAN, and the rectangular grid is both a CAR and the equivalent distorted TAN. This polynomial "correction" plus the plane-to-plane transform is still much faster than the normal reprojection. While this still does not cover all the possible transformations, it does include all those used for very large data collections.

\subsection{Performance Improvements Through Parallel Processing}

A simple way of improving performance is to run Montage on multiprocessor systems or cluster computers, now relatively inexpensive. Many parts of the Montage processing can be run in parallel. This is shown in Figure 3, which shows a parallelized processing flow for the simple case of processing three input images. Reprojection of input images can obviously be run on as many processors as are available, but many of the background rectification operations can be run in parallel too. Fitting planes to the overlap between pairs of images can be performed in parallel as soon as the reprojection of both images has been completed. Calculation of the parameters of the best-fit background model requires that all the overlap fitting has been completed, but subsequent application of the model to the individual images can be done in parallel.

The Montage project has been developing a parallel processing architecture for Montage that takes advantage of the parallelization inherent in the design and is intended to run on all types of parallel processing environments, including computing grids, clusters and supercomputers. Thus far, the architecture has been run successfully on Condor-pools, LSF and PBS managed clusters, and the Teragrid (through PBS).

\subsubsection{The Montage TeraGrid Portal}

The Montage TeraGrid portal has a distributed architecture, as illustrated in Figure 4. The portal is comprised of the following five main components, each having a client and server: (i) User Portal, (ii) Abstract Workflow Service, (iii) 2MASS Image List Service, (iv) Grid Scheduling and Execution Service, and (v) User Notification Service. These components are described in more detail below. 


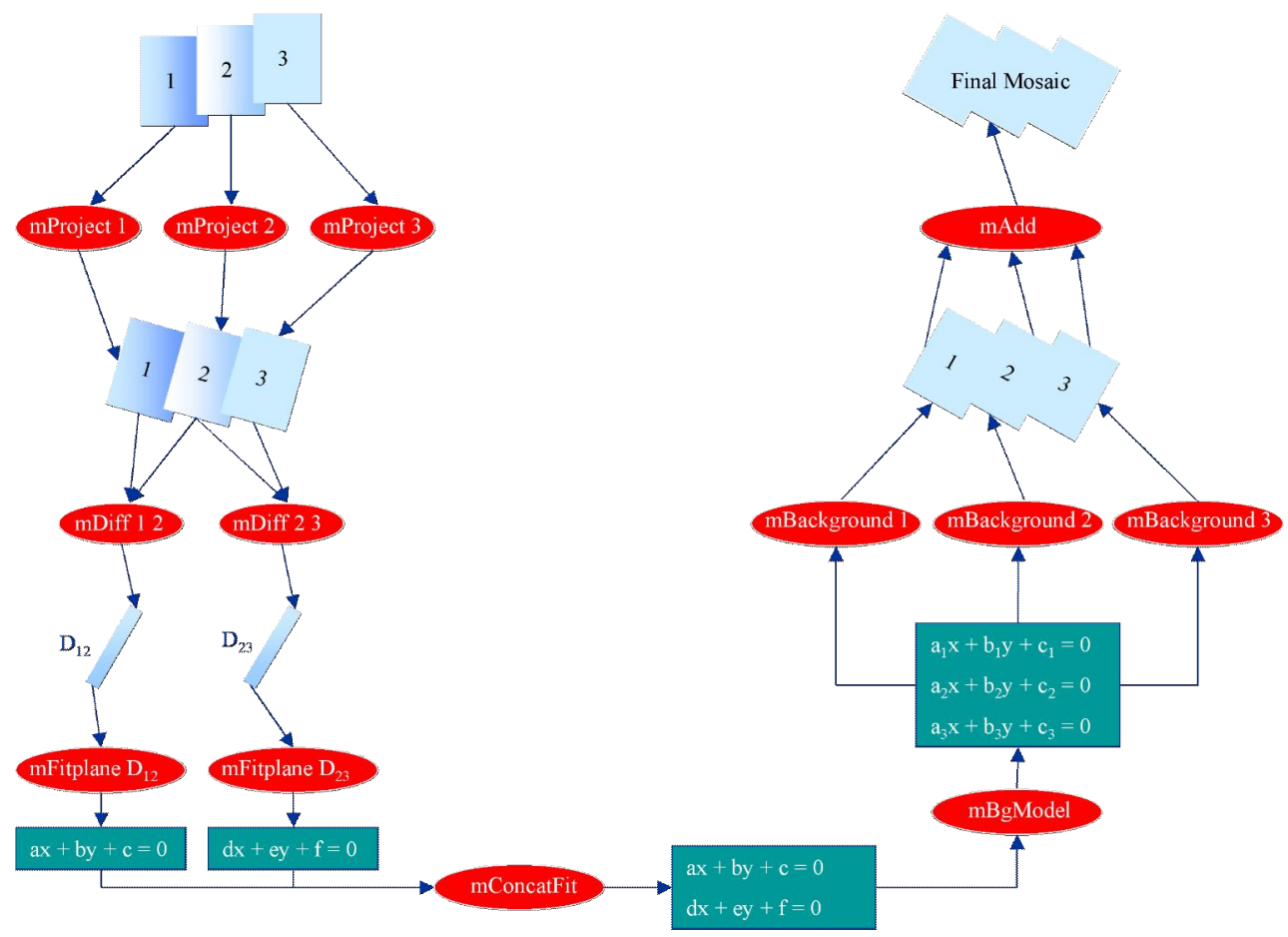

Figure 3: Example of Parallel Processing Workflow in Montage, for a case when three images are to be made into a mosaic. The reprojection, calculation of the inter-image differences and application of the background rectification can all be performed in parallel.

A usage scenario is as follows. Users on the internet submit mosaic requests by filling in a simple web form with parameters that describe the mosaic to be constructed, including an object name or location, mosaic size, coordinate system, projection, and spatial sampling. A service at JPL/Caltech is contacted to generate an abstract workflow, which specifies: the processing jobs to be executed; input, output, and intermediate files to be read or written during the processing; and dependencies between the jobs. A 2MASS image list service at IPAC/Caltech is contacted to generate a list of the 2MASS images required to fulfill the mosaic request. The abstract workflow identifies all the computations and files in a logical form without identifying specific resources that are needed to execute the workflow or the specific data locations. The abstract workflow is passed to a service at the Information Sciences Institute (ISI), University of Southern California, which runs software called Pegasus to schedule the workflow [12,13]. Pegasus maps the abstract workflow to an executable form and schedules the workflow on the Teragrid. The resulting "concrete workflow" includes information about specific file locations on the grid and specific grid computers to be used for the processing. The workflow is then executed on the remote TeraGrid clusters using Condor's DAGMan $[14,15]$. The last step in the mosaic processing is to contact a user notification service at IPAC/Caltech, which currently simply sends an email to the user with the URL of the Montage output.

This design exploits the parallelization inherent in the Montage architecture[ACL4]. The Montage grid portal is flexible enough to run a mosaic job on a number of different cluster and grid computing environments, including Condor pools and TeraGrid clusters. We have demonstrated processing on both a single cluster configuration and on multiple clusters at different sites having no shared disk storage.

User Portal: Users on the internet submit mosaic requests by filling in a simple web form with parameters that describe the mosaic to be constructed, including an object name or location, mosaic size, coordinate system, projection, and spatial sampling. After request submission, the remainder of the data access and mosaic processing is fully automated with no user intervention. 


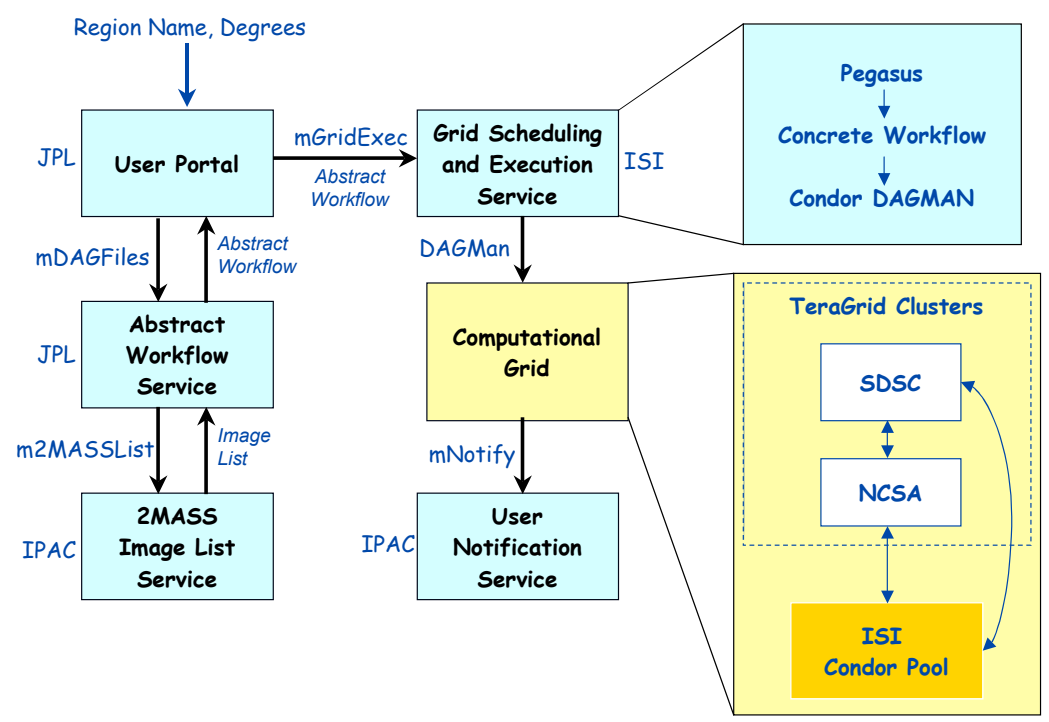

Figure 4: The distributed architecture of the Montage TeraGrid Portal.

The server side of the user portal includes a CGI program that receives the user input via the web server, checks that all values are valid, and stores the validated requests to disk for later processing. A separate daemon program with no direct connection to the web server runs continuously to process incoming mosaic requests. The processing for a request is done in two main steps:

1. Call the abstract workflow service client code

2. Call the grid scheduling and execution service client code and pass to it the output from the abstract workflow service client code

Abstract Workflow Service. The abstract workflow service takes as input a celestial object name or location on the sky and a mosaic size, and returns a zip archive file containing the abstract workflow as a directed acyclic graph (DAG) in XML and a number of input files needed at various stages of the Montage mosaic processing. The abstract workflow specifies the jobs and files to be encountered during the mosaic processing, and the dependencies between the jobs. These dependencies are used to determine which jobs can be run in parallel on multiprocessor systems. Figure 3, described earlier, is a pictorial representation of an abstract workflow for a mosaic with three input images.

2MASS Image List Service. The 2MASS Image List Service takes as input a celestial object name or location on the sky (which must be specified as a single argument string) and a mosaic size. The 2MASS images that intersect the specified location on the sky are returned in a table, with columns that include the filenames and other attributes associated with the images.

Grid Scheduling and Execution Service. The Grid Scheduling and Execution Service takes as input the zip archive generated by the Abstract Workflow Service, which contains the abstract workflow, and all of the input files needed to construct the mosaic. The service authenticates users, schedules the job on the grid using a program called Pegasus, and then executes the workflow using Condor's DAGMan.

Users are authenticated on the TeraGrid using their Grid security credentials. The user first needs to save their proxy credential in the MyProxy server [16]. MyProxy is a credential repository for the Grid that allows a trusted server (like our Grid Scheduling and Execution Service) to access grid credentials on behalf of a user. This allows these credentials to be retrieved by the portal using the user's username and password.

Once authentication is completed, Pegasus schedules the Montage workflow onto the TeraGrid or other clusters managed by PBS, Condor, LSF and others. Pegasus is a workflow management system designed to map abstract 
workflows onto the grid resources to produce concrete (executable) workflows. The Metadata Catalog Service (MCS) [17] keeps track of the mosaics produced. Pegasus consults various Grid information services, such as the Globus Monitoring and Discovery Service (MDS) [18] to discover which compute resources are available, the Globus Replica Location Service (RLS) [19] to discover data locations, and the Transformation Catalog [20] to determine where the Montage executable images are installed. Pegasus reduces the abstract workflow by pruning those workflow products that are already registered in the RLS. The executable workflow generated by Pegasus specifies the grid computers to be used, the data movement for staging data in and out of the computation, and the data products to be registered in the RLS and MCS.

The executable workflow is submitted to Condor's DAGMan for execution. DAGMan is a scheduler that submits jobs to Condo-G in the order specified by the concrete workflow. Condor-G queues the jobs for execution on the TeraGrid. Upon completion, the final mosaic is delivered to a user-specified location, and the User Notification Service, described below, is contacted.

User Notification Service. The last step in the grid processing is to notify the user of the URL where the mosaic may be downloaded. This notification is performed by a remote user notification service at Caltech, IPAC, so that a new notification mechanism can be used later without having to modify the Grid Scheduling and Execution Service. Currently the user notification is performed with a simple email, but a later version will use the Request Object Management Environment (ROME), being developed separately for the National Virtual Observatory. ROME will extend our portal with more sophisticated job monitoring, query, and notification capabilities.

\subsubsection{Performance of the Montage Teragrid Portal}

Table 1 gives the runtimes of the individual workflow components to summarize the results of running a 2-degree M16 mosaic on the NCSA TeraGrid cluster. These performance figures apply to the processing modules in version 1.7.x of Montage, which does not include the algorithmic optimizations described in Sections 2.3 and 2.4 The total runtime of the workflow was 107 minutes, and the workflow contained 1,515 individual jobs.

TABLE 1: TERAGRID PERFORMANCE OF MONTAGE

\begin{tabular}{l|l|l}
$\begin{array}{c}\text { Number } \\
\text { of Jobs }\end{array}$ & \multicolumn{1}{|c|}{ Job Name } & \multicolumn{1}{c}{ Average Run-Time } \\
\hline 1 & mAdd & 94.00 seconds \\
\hline 180 & mBackground & 2.64 seconds \\
\hline 1 & mBgModel & 11 seconds \\
\hline 1 & mConcatFit & 9 seconds \\
\hline 482 & mDiff & 2.89 seconds \\
\hline 483 & mFitplane & 2.55 seconds \\
\hline 180 & mProject & 130.52 seconds \\
\hline 183 & Transfer of data in & Between 5-30 seconds each \\
\hline 1 & Transfer of mosaic out & $18: 03$ minutes
\end{tabular}

Our main goal was to demonstrate the feasibility of running the Montage workflow in an automated fashion on the TeraGrid with some amount of performance improvement over the sequential version. Currently, Pegasus schedules the workflow as a set of small jobs. As seen in the table, some of these jobs run in only a few seconds, which is suboptimal because scheduling too many little jobs suffers from large overheads. These overheads stem from the resource schedulers that queue incoming jobs and the time that a job spends in the queue. In fact, if this processing was run on a single TeraGrid processor, it would have taken 445 minutes, so we are not taking very much advantage of the TeraGrid's parallelism. However, initially structuring the workflow in this way allows us to expose the highest degree of parallelism. 
We will improve this performance by optimizing both the Montage algorithms and the grid scheduling techniques. We expect about a 30 times speedup without sacrificing accuracy by using the algorithmic techniques described in Section 2.4. We will address the Teragrid performance in three ways: making Pegasus aggregate nodes in the workflow in a way that would reduce the overheads for given target systems; encouraging the Condor developers to reduce the per-job overhead; and examining alternate methods for distributing the work on the grid. Each option has advantages and disadvantages that will be weighed as we go forward.

\section{SCIENTIFIC APPLICATIONS}

The Montage software is available through a free, "clickwrap" license issued by the California Institute of Technology. Version 1.7 is publicly accessible, and Version 2.0 is currently available for evaluation on a shared risk basis. The software is finding broad applicability in astronomical research and in Education and Public Outreach.

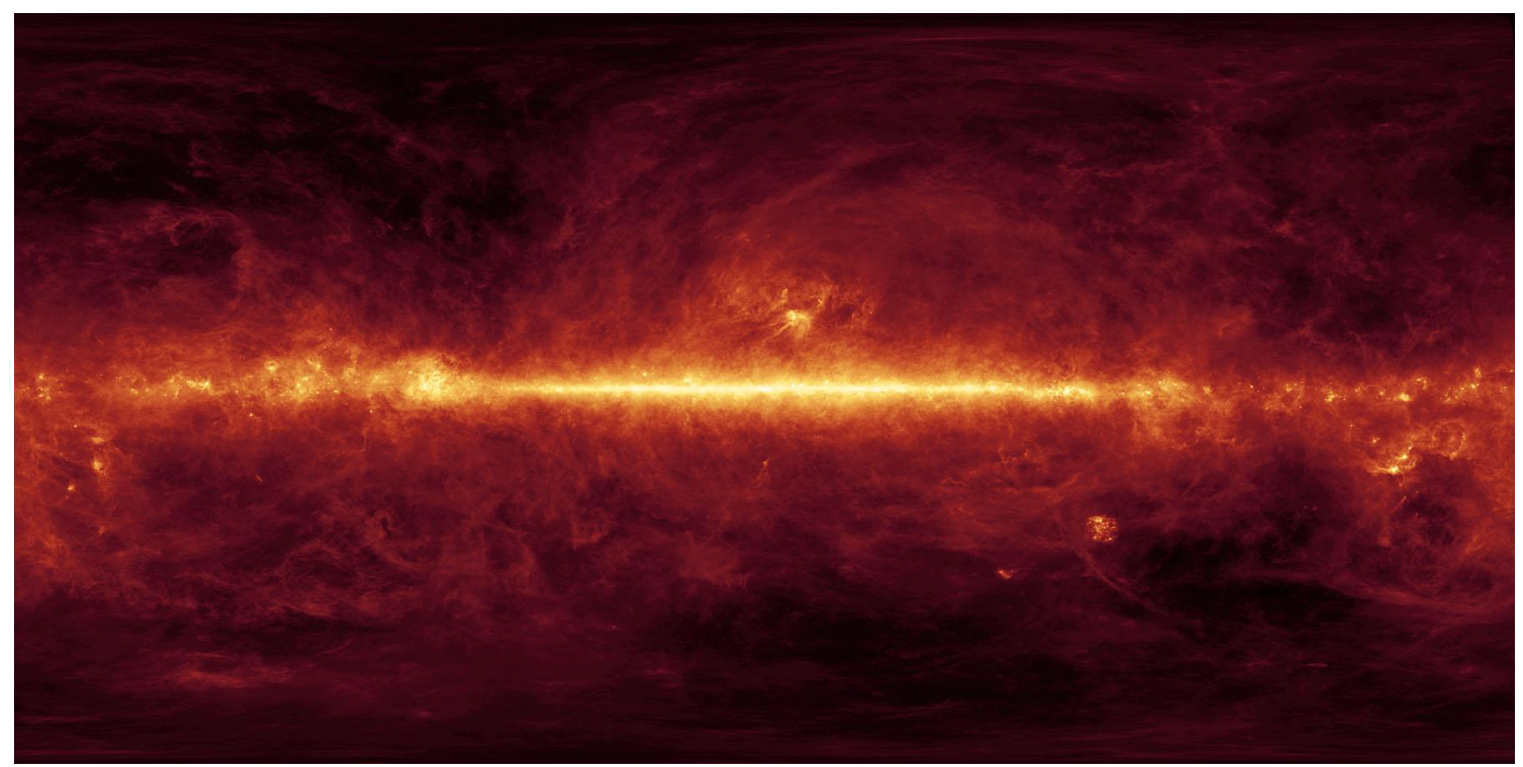

Figure 5: The $100 \mathrm{~m}$ sky represented in Cartesian projection, computed by Montage from composite DIRBE and IRAS skymaps of Schlegel, Finkbeiner and Davis (1998)

One application of Montage is as a general reprojection engine to derive large scale or full sky images. Figure 5 shows an image of the $100 \mathrm{~m}$ map of the sky by Schlegel, Finkbeiner and Davis (1998) [21] that aggregates the skymaps produced by the Diffuse Infrared Background Experiment (DIRBE), aboard the Cosmic Background Explorer (COBE), and the Infrared Astronomical Satellite (IRAS), shown transformed from the Zenithal Equal Area projection to the Cartesian projection. This map is a science product that can be made accessible to astronomers on-line, either as a single file for download or through a cut-out web service, which will deliver image subsets of arbitrary size centered on a target position. The NASA/Infrared Processing and Analysis Center (IPAC) Infrared Science Archive (IRSA) is including this image as part of a broader science service that is required by the Herschel mission for observation planning. It will return estimates of the dust emission, galactic emission and extinction along a line of sight, and when fully developed, will return fluxes extrapolated to other wavelengths. The Spitzer/IPAC E/PO group is planning to deliver E/PO products made from such mosaics, including fold-out icosahedrons of the sky that will be distributed on-line. 


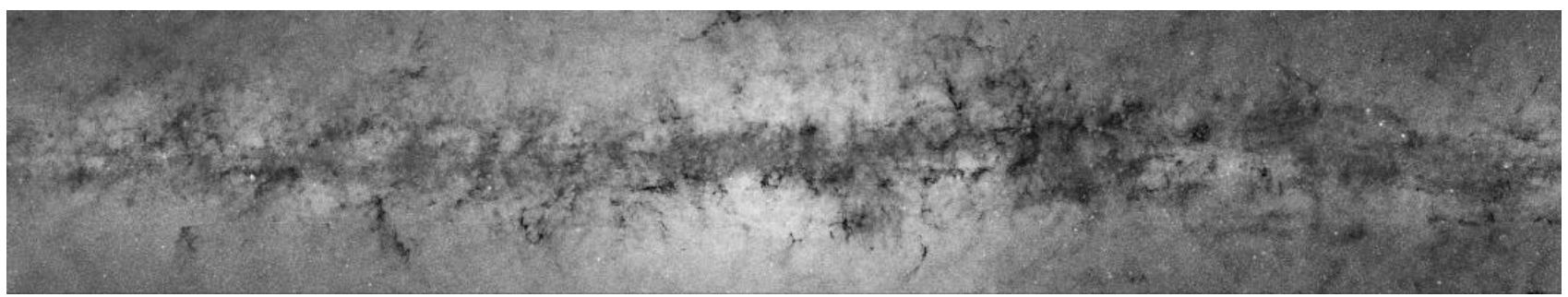

Figure 6: A section of the Galactic plane, $44^{\circ}$ by $8^{\circ}$, measured by 2 MASS in the J-band and shown in Cartesian projection. The full resolution image contains 4800 Mpixels.

A second application is generation of large scale image mosaics, which can also be served as products, either for download or through spatial subsetting services. Figure 6 shows a mosaic of a section of the galactic plane in the 2MASS J-band [5], $44 \mathrm{deg}$ long and $8 \mathrm{deg}$ wide, centered on the Galactic Center and shown in Cartesian projection. The production of this mosaic was intended as a pilot project to provide resource estimates for generation of a full-sky 2MASS mosaic, to be computed on the San Diego Supercomputer Center's IBM DataStar supercomputer, when fully commissioned. The mosaic was produced on a cluster of four $1.4 \mathrm{Ghz}$ Linux processors that processed the input images in parallel. By taking advantage of the algorithmic improvements described in Section 2.3 and 2.4 , the map was generated in 4-hours wall clock time from 16,000 2MASS images in sine projection and containing $512 \times 1024$ pixels each.

Montage has found particular application to the Spitzer Space Telescope, and this is described in the remainder of this section.

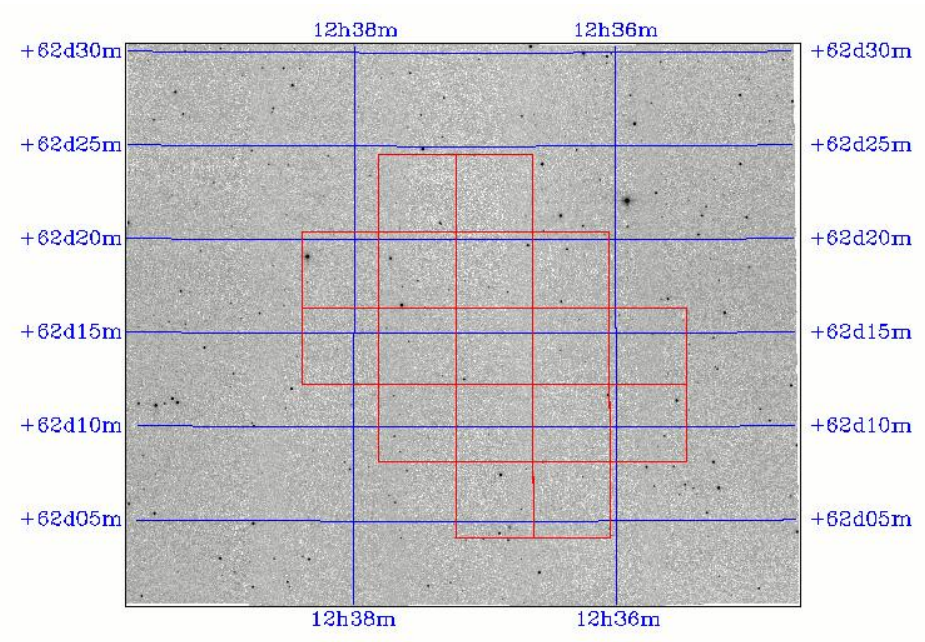

Figure 7: Footprints of Hubble Space Telescope (HST) Advanced Camera System (ACS) images in the Hubble Deep Field North supporting the Great Observatories Origins Deep Survey (GOODS), shown on a backdrop 2MASS K-band mosaic computed with Montage. The GOODS data query service of which this is a part is accessible at http://irsa.ipac.caltech.edu/data/GOODS

Backdrops for Rendering Spatial Coverage of Spitzer Ancillary Observations. On behalf of the Spitzer Science Center, IRSA is serving ancillary, ground based data supporting the Spitzer First Look Survey and Legacy projects. The data generally consist of collections of images, spectra and source catalogs covering target areas that are generally several degrees on a side. These targets include the Lockman Hole, the ELAIS Fields and the Hubble Deep Fields. Montage has been used to create mosaics, generally from 2MASS All-Sky images, that act as background images that render the sky coverage of results of spatial searches for data. An example is shown above in Figure 7.

Support for Data Production and Validation by Spitzer Space Telescope Legacy Teams. Two Spitzer Legacy teams, the Spitzer Wide-area InfraRed Extragalactic (SWIRE) survey [22] and the Galactic Legacy Infrared Mid-Plane Survey Extraordinaire (GLIMPSE) [23], are actively using Montage to support their processing pipelines, quality assurance and 
mission planning. SWIRE will use the Infrared Array Camera and MIPS to trace the evolution of extragalactic populations at moderate redshift. GLIMPSE plans to generate a multi-wavelength infrared atlas of the Galactic Plane.

SWIRE has used Montage on Solaris platforms as a fast reprojection and co-addition engine to build sky simulations at a common spatial sampling that model the expected behavior of the sky, including galaxies, stars and cirrus. These simulations have been used to validate the processing pipeline and source extraction. Predictions of the expected source populations and appearance of the sky have been used to plan the observing strategy. Following the launch of Spitzer, SWIRE has applied Montage to the production of science products. Figure 8 shows part of a 2.5 GB mosaic generated from images obtained with Spitzer's Infrared Array Camera (IRAC); the bright galaxy left of center is the Tadpole Galaxy. Montage was used here as a background rectification and co-addition engine, applied to mosaic images generated as part of the Spitzer pipeline.

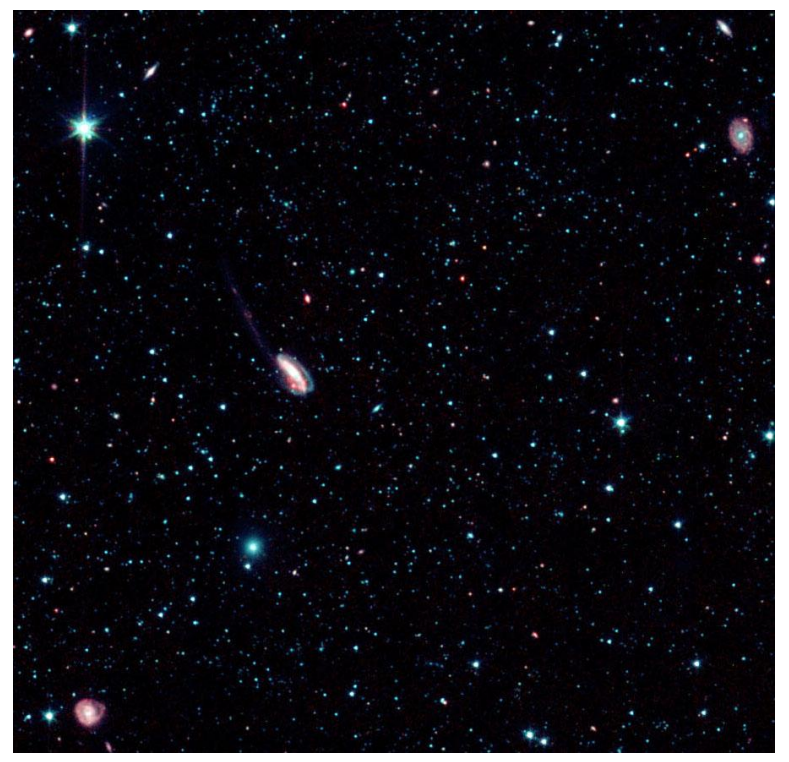

Figure 8: Part of a three color mosaic of Spitzer Infrared Array Camera (IRAC) images. The complete mosaic is 10,000 pixels on a side.

The GLIMPSE team has also integrated Montage into their Linux cluster based pipeline. As part of their quality assurance program, they have used mosaics of the entire GLIMPSE survey region at J, H, K and MSX $8 \mathrm{~m} \mathrm{[24].} \mathrm{This}$ will provide quicklook comparisons for quality assurance of the IRAC mosaics

\section{ACKNOWLEDGEMENTS}

Montage is supported by the NASA Earth Sciences Technology Office Computing Technologies program, under Cooperative Agreement Notice NCC 5-6261. Pegasus is supported by NSF under grants ITR-0086044 (GriPhyN) and ITR AST0122449 (NVO).

Part of this research was carried out at the Jet Propulsion Laboratory, California Institute of Technology, under a contract with the National Aeronautics and Space Administration. Reference herein to any specific commercial product, process, or service by trade name, trademark, manufacturer, or otherwise, does not constitute or imply its endorsement by the United States Government or the Jet Propulsion Laboratory, California Institute of Technology. 


\section{REFERENCES}

1. The Montage project web page, http://montage.ipac.caltech.edu/

2. The Flexible Image Transport System (FITS), http://fits.gsfc.nasa.gov, http://www.cv.nrao.edu/fits.

3. E.W. Greisen and M. Calabretta, Representation of Celestial Coordinates In FITS, http://www.atnf.csiro.au/people/mcalabre/WCS.htm.

4. The Distributed Terascale facility, http://www.teragrid.org/

5. The 2MASS Project, http://www.ipac.caltech.edu/2mass

6. The Digitized Palomar Observatory Sky Survey (DPOSS), http://www.astro.caltech.edu/ george/dposs

7. The Sloan Digital Sky Survey, http://www.sdss.org/

8. Berriman, G.B., Curkendall, D., Good, J., Joseph, J., Kataz, D. S., Kong, Monkewitz, S., Moore, R., Prince, T., and Williams, R. 2002. "An Architecture for Access to a Compute Intensive Image Mosaic Service in the NVO." In Virtual Observatories, A S. Szalay, ed. Proceedings of SPIE, Vol 4686, 91.

9. Montage Version 1.7.x documentation and download http://montage.ipac.caltech.edu/docs/

10. Montage Version 1.7.x. Photometric and Calibration Accuracy, http://montage.ipac.caltech.edu/docs/accuracy.html

11. Mopex, the Spitzer Science Center Mosaic Engine, http://ssc.spitzer.caltech.edu/postbcd/doc/mosaicer.pdf

12. E. Deelman, J. Blythe, Y. Gil, C. Kesselman, G. Mehta, S. Patil, M.-H. Su, K. Vahi, M. Livny, Pegasus: Mapping Scientific Workflows onto the Grid, Across Grids Conference 2004, Nicosia, Cyprus

13. Y. Gil, E. Deelman, J. Blythe, C. Kesselman, and H. Tangmurarunkit, Artificial Intelligence and Grids: Workflow Planning and Beyond, IEEE Intelligent Systems, January 2004

14. Condor Team. 2002. The Directed Acyclical Graph Manager, http://www.cs.wisc.edu/condor/dagman. 2002.

15. Frey, J., Tannenbaum, T., Foster, I., Livny, M., and Tuecke, S. 2002. Cluster Computing, 5, 237.

16. Novotny, J. et al. 2001. "An On-Line Credential Repository for the Grid: MyProxy." Proceedings of the $10^{\text {th }}$ IEEE International Symposium on High Performance Distributed Computing

17. Deelman, E. et al. 2001. Technical Report GriPhynN 2001-17

18. Czajkowski, K. et al. 2001. "Grid Information Services for Distributed Resource Sharing." Proceedings of the $10^{\text {th }}$ IEEE International Symposium on High Performance Distributed Computing

19. Chervenak, A. 2002. "Giggle: A Framework for Constructing Scaleable Replica Location Services." Proceedings of Supercomputing 2002.

20. Singh, G. et al. 2003. "A Metadata Catalog Service for Data Intensive Applications." Proceedings of Supercomputing 2003

21. Schlegel, D. J, Finkbeiner, D.P, and Davis, M. 1998, Ap. J., 500, 525

22. Lonsdale, C. J. et al. 2003. Pub Ast Soc Pac, 115, 897

23. The Galactic Legacy Infrared Mid-Plane Survey Extraordinaire (GLIMPSE), http://www.astro.wisc.edu/sirtf/

24. GLIMPSE Validation Images, http://www.astro.wisc.edu/sirtf/2massimages/2massimages.html 